\title{
A green chemistry perspective on catalytic amide bond formation
}

\author{
Marco T. Sabatini, ${ }^{1}$ Lee. T. Boulton, ${ }^{2}$ Helen F. Sneddon, ${ }^{2}$ Tom D. Sheppard ${ }^{1 *}$
}

${ }^{1}$ Department of Chemistry, University College London, WC1H OAJ, UK; ${ }^{2}$ GSK, Medicines Research Centre, Gunnels Wood Road, Stevenage, SG1 2NY, UK. *Email: tom.sheppard@ucl.ac.uk

Abstract: The synthesis of amides is of widespread importance, and there has been considerable recent interest in the development of catalytic methods to access these molecules. In this perspective article, we provide an overview of the current state-of-the-art of amide synthesis, and assess new catalytic amide formation methods in the context of efficiency and sustainability. The advantages and disadvantages of catalytic approaches are highlighted and areas for future research are identified.

\section{Introduction}

Amides are an extremely important class of organic molecules that find application in a wide range of fields, as well as serving as useful synthetic intermediates for accessing other key compound classes, including complex amines and heterocycles. Consequently, the synthesis of amides is one of the most widespread processes in organic chemistry research laboratories. Industrially, the application of amide synthesis is also commonplace, from bulk chemical and polymer synthesis, to agrochemical and pharmaceutical production.

Amidation Methods. Despite the ubiquity of amide synthesis in both small and large-scale processes, commonly used approaches suffer from many drawbacks. Typical methods for amide bond formation employ stoichiometric quantities of activating reagents such as EDC (1-Ethyl-3-(3-dimethylaminopropyl)carbodiimide), HATU (: (1-

[Bis(dimethylamino)methylene]-1H-1,2,3-triazolo[4,5-b]pyridinium 3-oxide hexafluorophosphate), thionyl chloride, or T3P ( $n$-propylphosphonic acid anhydride) ${ }^{1}$. which lead to the production of large quantities of waste. It should be noted however that the frequency of usage of such methods changes significantly with increasing scale; in a process chemistry setting the use of HATU and related coupling reagents is less common, with lower cost, more stable and lower molecular weight reagents such as CDI (1,1'Carbonyldiimidazole), thionyl chloride, oxalyl chloride, and T3P being more widely used ${ }^{2}$. There has been a significant move towards the adoption of green chemistry in both academic and industrial settings in recent years. Indeed, the green chemistry movement has gained significant traction with the development of the "twelve principles of green chemistry" 3 and the subsequent introduction of metrics for comparing the efficiency of chemical processes $^{4}$. In 2005, the American Chemical Society Green Chemistry Institute Pharmaceutical Roundtable (ACS GCIPR) was formed with the aim of 'encouraging innovation while catalysing the integration of green chemistry into the pharmaceutical industry ${ }^{5}$. As the most heavily utilised transformation in the pharmaceutical sector ${ }^{6}$, it was 
perhaps fitting that amide bond formation avoiding poor atom economy reagents was voted as the top challenge by the ACS GCIPR in $2007^{5}$. This has served to stimulate renewed interest in new methodologies for amide bond formation, driven by the low efficiency and poor sustainability of current methods, especially on large scale ${ }^{5}$, and there is certainly considerable scope for improving the green credentials of these reactions through innovative approaches.

Solvents. The solvent employed in an amide coupling reaction is also a very important factor. Dipolar aprotic solvents [such as NMP ( $N$-methyl-2-pyrrolidinone), DMF $(N, N$ dimethylformamide) and DMAc ( $N, N$-dimethylacetamide)], have reprotoxicity concerns, and are subject to $\left(\mathrm{NMP}^{7}\right)$, or may in future face, increasing regulation in Europe. Chlorinated solvents such as $\mathrm{CH}_{2} \mathrm{Cl}_{2}$, which are common alternatives, can also present safety issues ${ }^{8}$, alongside increased costs associated with their disposal. It is worth noting that from a pharmaceutical industry perspective, there is considerable merit in promoting the use of less harmful solvents ${ }^{9}$ in the discovery phase as small-scale synthetic procedures developed there are often directly employed in the early stages of compound development. Embedding the use of green chemistry principles at an early stage can therefore help to significantly increase sustainability within pharmaceutical development.

Catalytic amide synthesis. Despite a recent surge in the development of catalytic methodologies for amide bond formation in the past two decades, the uptake of these methodologies has been extremely low. We hypothesise here that this may be partly down to a limited reaction scope, but also because they do not typically satisfy many key green chemistry criteria. The development of new amidation methods should take into account the very high bar already set by existing processes employing stoichiometric reagents, in terms of overall effectiveness (reaction yield, purity), waste generation and practicality. This review aims to assess the current state of the field of amide bond formation, and place recent developments in perspective, with an overarching goal of directing future research in catalytic amidation that can lead to tangible improvements in terms of sustainability. Fundamentally, this requires syntheses to have "greater energy efficiency, selectivity, simplicity, and safety for nature and human health"10. Our evaluation will therefore consider aspects relating to effectiveness, safety, cost, efficiency, and practicality (Figure 1a).

\section{Strategies for amide synthesis}

Strategies for amide synthesis are outlined in Figure 1b. The vast majority of amides are currently synthesised via a condensation reaction between a carboxylic acid and amine, mediated by a stoichiometric activating agent (A). These activating agents are typically of relatively high molecular weight (Table), especially given the fact that the condensation 
reaction only requires removal of a molecule of water (molecular weight 18). In some cases, a direct thermal condensation reaction can be achieved (B), but in practice this approach is rarely employed except for simple substrates. A potentially ideal approach is the use of a catalyst for mediating direct amidation under milder conditions (C), and recent research has shown that both boron-based catalysts and those derived from group(IV) metals can be particularly effective. Whilst the focus of this article will be on direct condensation reactions of carboxylic acids and amines, as this approach is able to take advantage of the widespread availability of these common reagents, alternative catalytic strategies such as amidation of esters (D), oxidative amidation of primary alcohols or aldehydes (E), and carbonylative amidation of aryl halides $(\mathbf{F})$, are gaining ground as viable routes to particular classes of amide.

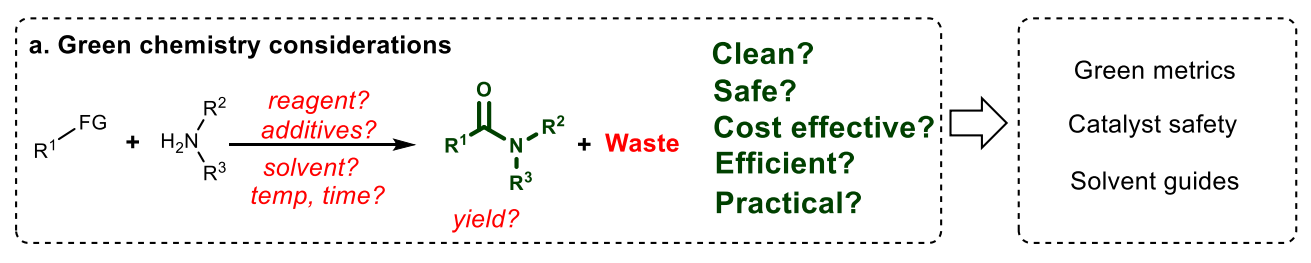

b. Common amidation approaches \& new developments

(B) Thermal amidation

(C) Catalytic condensation

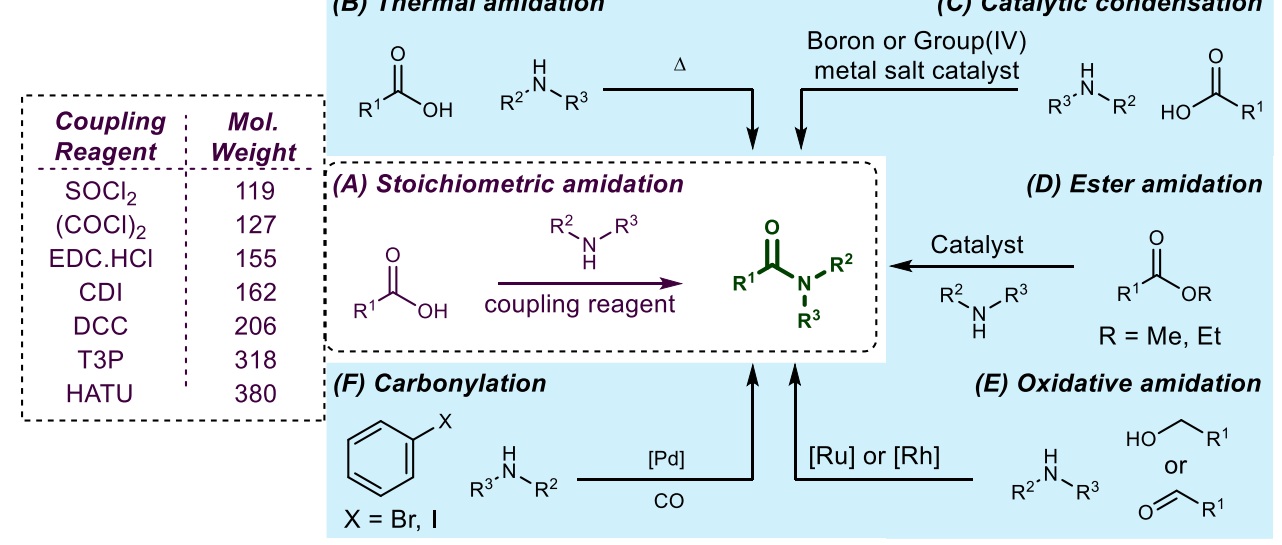

Figure 1: Methods for Amide Synthesis a) Green chemistry considerations. b) Common stoichiometric amide coupling reagents and their molecular weights, and the catalytic amidation approaches considered in this article. Abbreviations: EDC: 1-Ethyl-3-(3dimethylaminopropyl)carbodiimide; CDI: Carbonyldiimidazole; DCC:

Dicyclohexylcarbodiimide; T3P: n-propylphosphonic acid anhydride; HATU: (1-

[Bis(dimethylamino)methylene]-1H-1,2,3-triazolo[4,5-b]pyridinium 3-oxide hexafluorophosphate

\section{Current state of the art}

As noted above, catalytic amidation reactions are rarely used preparatively, even though it is more than 20 years since the first report of boronic acid catalysed direct amidation ${ }^{11}$. It is constructive at this point, to consider which amide formation methods are most prevalent, 
both in the wider research community, and in the context of larger scale pharmaceutical preparation. A search of the chemical literature in the Reaxys database from 2017-2018 (Figure 2a, Supplementary Data) revealed, perhaps unsurprisingly, that coupling reagents are the go-to approach for most researchers, with carbodiimides (EDC, DCC) and uronium salts (HATU, HBTU) accounting for around $45 \%$ of the amidation reactions reported, and with CDI and T3P being employed in a further $4 \%$ of cases. The other common approach is acid chloride formation, using one of the common low-cost chlorinating agents (thionyl chloride, oxalyl chloride) which was employed in $\sim 16 \%$ of cases. We also examined the most common solvents used in these amidation reactions, with DMF and $\mathrm{CH}_{2} \mathrm{Cl}_{2}$ proving most prevalent, even though it has been demonstrated that many amide coupling reactions can be achieved in less-hazardous solvents ${ }^{12-13}$. The widespread use of these solvents for amidation is probably down to their ability to solubilise reactants effectively, but historical usage undoubtedly plays a part in their continued popularity.

In order to provide an insight into common approaches to larger scale amidation reactions, we examined data for multigram (typically $>300 \mathrm{~g}$ scale) amidation reactions operated within GSK in 2016 (Figure 2b, Supplementary Data). In these larger scale reactions, the quantity of waste produced becomes more significant, and this is reflected in the higher prevalence of reactions using either a commercial acid chloride as the acylating reagent, or the generation of an acid chloride in situ using a low-cost chlorinating agent (32\%). Amide coupling reagents are also used in a significant proportion of reactions (45\%), but on these larger scales reagents such as T3P (19\%) and CDI (7\%) are usually preferred, though HATU was still also used (11\%). The formation of a mixed anhydride is also a common low-cost approach to carboxylic acid activation (16\%). Interestingly, in a small number of processes thermal amidation was employed, although this was only with relatively facile amide formations (e.g. lactam formation; formamide synthesis). There were no examples of catalytic amidations, and in general the reagent use is not dissimilar to that in an analysis of drug candidate syntheses across three pharmaceutical companies reported by Carey et al. in $2006^{14}$. Despite recent efforts to minimise usage earlier in the pipeline, $\mathrm{CH}_{2} \mathrm{Cl}_{2}$ was still the most common solvent used for amidation (though it should be noted that many if not all of these reactions are likely to see changes to a more benign solvent before transferring to pilot plant), but hazardous dipolar aprotic solvents (DMF) were much less prevalent than in the Reaxys dataset. Notably, five of the solvents used in these large-scale reactions were not commonly found in the Reaxys dataset (2-MeTHF, EtOAc, DMA, DMSO, IPrOAc). 
a) Amidation reactions found in the Reaxys database (2017-18, $\sim 40 \mathrm{k}$ reactions)
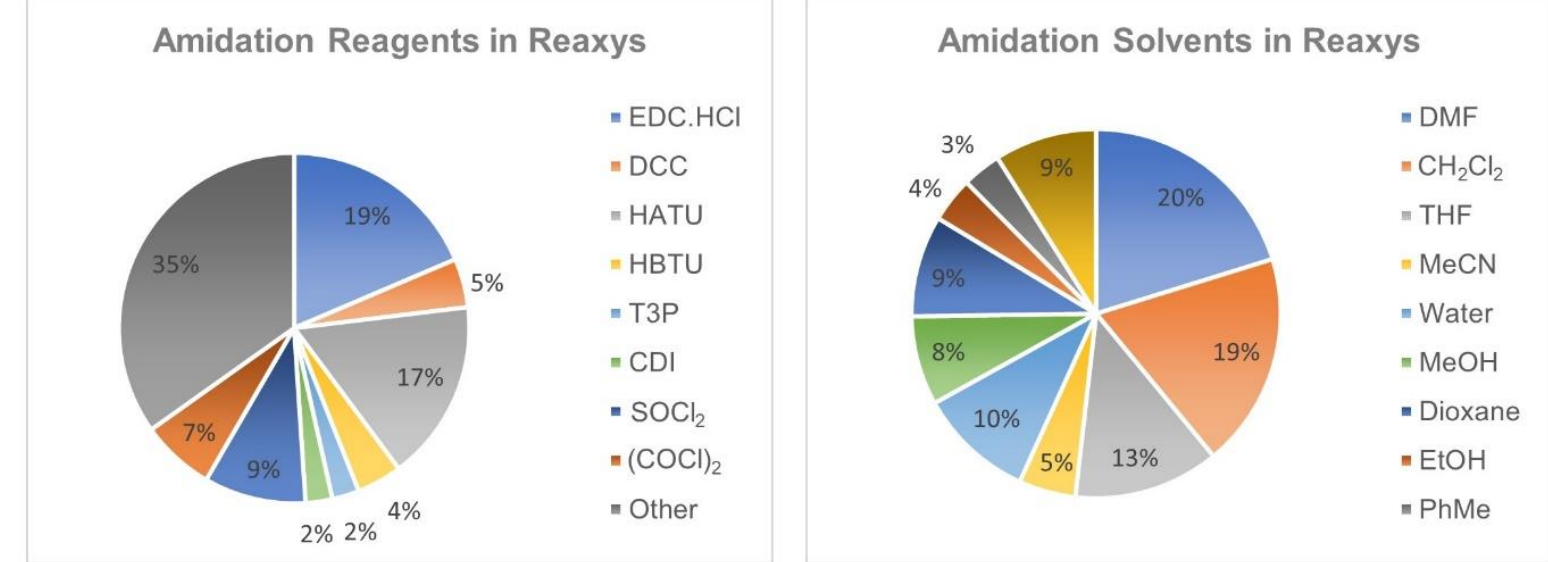

b) Large scale ( $>300 \mathrm{~g}$ ) amidation reactions within GSK in 2016 (56 reactions)

GSK Amidation Reagent Data

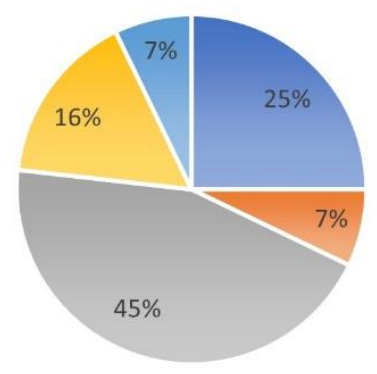

- Chlorinating Agent

- Acid chloride

= Coupling reagent

" Mixed anhydride

= Other

c) Typical PMI data

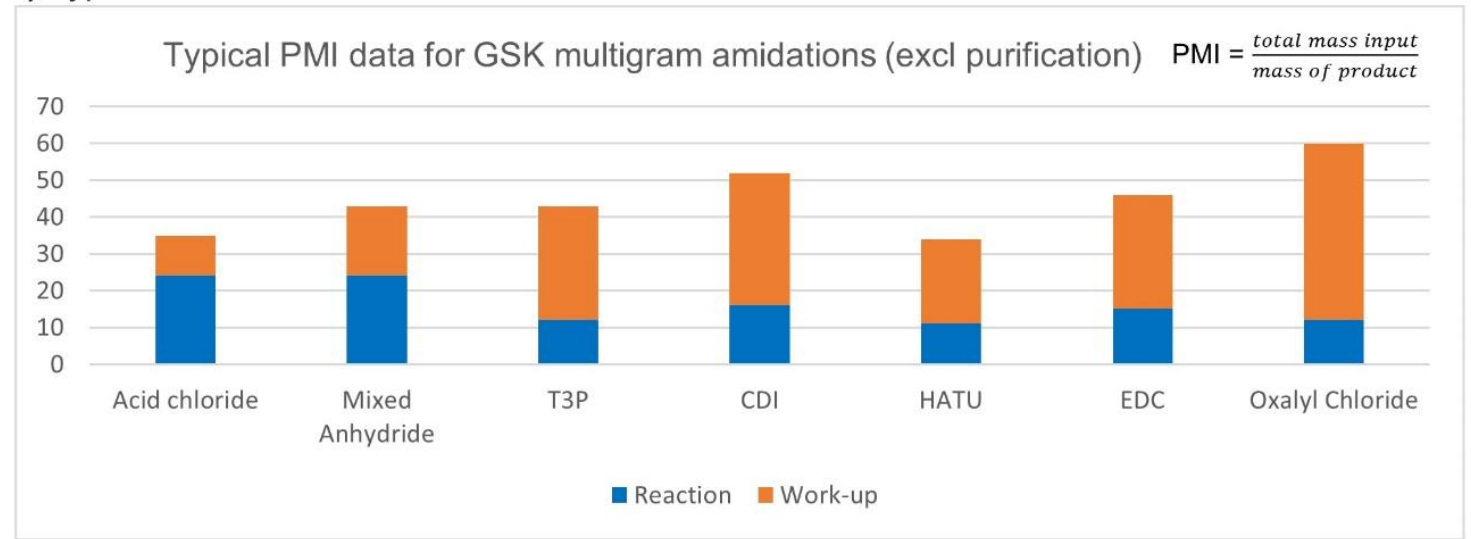

\section{Figure 2: Analysis of methods currently used for the preparation of amides a)}

Common methods used for amidation found on the Reaxys database (2017-18) showing the reagent and solvent used; b) Methods used for large-scale amidation within GSK showing the reaction-type and solvent used; c) Typical Process Mass Intensity ${ }^{4}$ (PMI) data for multigram amidation reactions at GSK using various methods (Average $\mathrm{PMI}=45$ ); data excludes purification steps (e.g. recrystallisation/chromatography). Abbreviations: HBTU: $N, N, N^{\prime}, N^{\prime}$-Tetramethyl-O-(1H-benzotriazol-1-yl)uronium hexafluorophosphate; DMF: $N, N$ dimethylformamide; THF: Tetrahydrofuran; MeTHF: 2-methyltetrahydrofuran; DMA: $N, N$ dimethylacetamide; DMSO: Dimethylsulfoxide; TBME: tert-butyl methyl ether. 
It is commonly assumed that a catalytic reaction must inherently be more efficient than one mediated by a stoichiometric reagent, and indeed the $9^{\text {th }}$ principle of green chemistry promotes the use of catalysts over reagents ${ }^{3}$. However, the majority of waste generated in a chemical reaction usually comes from the solvent requirements of the process, especially that used in reaction work-up and purification. Hence, a stoichiometric reagent may be more efficient if it can produce the product in greater yield and with higher purity. Process Mass Intensity $(\mathrm{PMI})^{4}$ is a widely used industry metric that provides a useful and directly comparable measure of the efficiency of a chemical reaction. PMI is calculated from the total material input (the mass of all reagents, catalysts, solvents, water, etc) required to synthesise each kilogram of product. Evaluation of large scale amidation data from GSK illustrates that stoichiometric amidation reactions using acid chlorides, mixed anhydrides or coupling reagents can often be achieved with very high efficiency (Figure 2c), with PMI values ranging from 30-60. Thus, newly developed catalytic amidation methodologies would have to demonstrate at least comparable scalability and efficiency to be competitive with these well-established approaches. As will be seen from the discussion below, in most cases researchers working in this area have simply not explored whether their newly developed reactions can be made more efficient on scale-up; in fact there are very few catalytic amidation reactions that have been demonstrated on even a $10 \mathrm{~g}$ scale. In contrast, some newly reported low-cost amidation reagents such as $\mathrm{Si}(\mathrm{OMe})_{4}$ have been demonstrated to be applicable to efficient large-scale amidation reactions (PMI 43, for a $158 \mathrm{~g}$ amide synthesis $)^{15}$. Similarly, amidation reactions using COMU [(1-Cyano-2-ethoxy-2oxoethylidenaminooxy)dimethylamino-morpholino-carbenium hexafluorophosphate] as a stoichiometric coupling agent have been demonstrated in aqueous micelles with very high efficiency though only on relatively small scale ${ }^{16}$.

For an amidation reaction to be suitable for large-scale pharmaceutical synthesis, fundamentally it must be a clean and high-yielding process from which the target amide can readily be obtained in high purity. The reagent/catalyst cost is also important, as are potential safety/toxicity issues of the reagent/catalyst (and associated byproducts). For example, the use of 1-hydroxybenzotriazole and its derivatives on large scale has fallen out of favour as a consequence of potential explosion hazards ${ }^{17}$.

\section{Catalytic direct amidation reactions}

The direct thermal condensation of a carboxylic acid and amine to give an amide is possible in some cases, and offers a potentially attractive approach, as very little waste is produced ${ }^{18}$. The major drawback of these reactions is the limited range of substrates which can be successfully employed. Although increasing the reaction temperature can help to facilitate 
more 'difficult' amidation reactions, in practice this can raise issues with regard to the thermal stability of complex substrates. Nevertheless, the reaction has been demonstrated to be effective for preparation of multigram quantities of amide with high efficiency (A multigram amidation reaction using simple substrates with a $\mathrm{PMI}$ of $<5$ has been reported ${ }^{18}$ ), and thermal amidation is already a viable option for 'less challenging' amide formations on process scale.

Catalytic direct amidation provides an opportunity to lower the energy requirements of the reaction, and to expand the substrate scope (Figure 3). All of the catalytic systems reported to-date require water-removal from the reaction mixture in order to operate effectively (Figure 3a). This can be achieved either by using excess molecular sieves in the reaction mixture, or by azeotropic water-removal (Dean-Stark conditions). The former is more common in the literature, and often enables the catalytic amidation process to operate at lower temperatures $\left(25-50^{\circ} \mathrm{C}\right)$. However, molecular sieves are impractical for large scale reactions, and even on a smaller scale can potentially lead to increased solvent requirements during both the reaction and work-up which impacts significantly on the efficiency. It should also be noted, that the process of adequately drying the molecular sieves prior to reaction set-up often requires a large energy input (high temperatures, vacuum), which may outweigh the benefits of being able to carry out the amidation reaction itself at lower temperature. There is certainly scope for further research into alternative strategies for water removal that add to the toolkit of methods available (e.g. biphasic/micellular conditions ${ }^{16}$, ultrafiltration, etc). The most successful catalysts to date include group(IV) metal salts ${ }^{18-19-20-21}$ and boron-based systems ${ }^{11,22-23-24-25-26-27-28-29-30}$ and these will both be evaluated in the context of sustainability. All of these elements (B, Ti, Zr) are relatively abundant and low-cost, and their compounds are generally considered to be non-toxic such that there are no general restrictions on their presence as trace impurities in pharmaceuticals. The catalysts employed range from very simple commercially available compounds to highly complex systems requiring multistep synthesis (Figure $3 b$ ). Readily available low-cost catalysts include boric acid \& borate esters, as well as simple $\mathrm{Ti} / \mathrm{Zr}$ compounds and boronic acids. More complex boron-derived catalysts including functionalised boronic acids and boron-containing heterocycles, which require several synthetic steps to prepare, have also been reported. Below, we will consider the advantages and drawbacks of each system with regard to reaction efficiency, substrate scope and safety issues.

Group(IV) metal catalysts. These catalysts were investigated independently by Williams ${ }^{18}$ and Adolffsson ${ }^{19}$, and, with the exception of hafnium derivatives, are produced from earthabundant metals ${ }^{31}$ and are available commercially at low-cost. Although it was shown to be 
less reactive than the zirconium catalysts, $\mathrm{Ti}\left(\mathrm{O}^{\prime} \mathrm{Pr}\right)_{4}$ is an inexpensive bulk chemical, and has perhaps been somewhat overlooked as an amidation catalyst since the original report ${ }^{20}$, especially given the fact that it is relatively non-hazardous. To date, no efficient large-scale application has been reported, even though it does show reasonable reactivity with functionalised substrates including amino acid derivatives ${ }^{20,24}$; no information regarding its reactivity with heterocycles/anilines has yet been published. $\mathrm{Cp}_{2} \mathrm{ZrCl}_{2}$ and $\mathrm{ZrCl}_{4}$ are reported to be more effective catalysts which have been applied to a wider substrate range (some anilines, but few heterocycles), and a reaction with improved efficiency has been

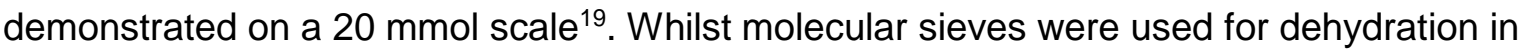
the vast majority of these reactions, azeotropic water removal has also been shown to be feasible using PhMe as solvent ${ }^{18,24}$. The corresponding hafnium catalyst ${ }^{21}$ did not offer any significant benefits over titanium or zirconium catalysts which could outweigh the increased cost of this relatively rare metal.

Boron-based catalysts. Boron compounds constitute the other important class of amidation catalysts, with boric acid being an extremely low-cost bulk chemical. An increasing concern in this area is the potential reproductive toxicity of boric $\operatorname{acid}^{32}$, which will ultimately be formed from breakdown of any of these boron-based catalysts. Nevertheless, boric acid is widespread in many existing consumer products, and stoichiometric boron reagents (e.g. boronic acids) are already widely used in the preparation of pharmaceuticals. The use of boric acid as an amidation catalyst ${ }^{22}$ has been demonstrated on large-scale, including in industrial processes ${ }^{33}$, and the reactions can be highly efficient when carried out with azeotropic water removal. The main drawback is the lack of catalyst activity with more challenging substrates (anilines, heterocyclics, amino acids). Commercially available borate esters can offer considerable improvements in reactivity and $\operatorname{scope}^{23}$, with many pharmaceutically relevant amides being prepared including those derived from poorly nucleophilic anilines, heterocyclic compounds, and amino acids (including unprotected amino acids ${ }^{24}$ ). These reactions can be scaled up to access multigram quantities of amide with very high efficiency (PMI from 5-13). The most commonly reported amidation catalysts are boronic acids, ranging from simple examples (phenyl, $n$-butyl), to more complex functionalised systems ${ }^{11,25-29}$. Each system has been reported to offer particular advantages, from lower temperature reactions, to wider substrate tolerance or increased reaction rate. However, the reactions are all broadly similar, employing molecular sieves (in the reaction flask itself for lower temperature examples) or azeotropic water removal. Significant limitations in reaction scope remain however, with few examples of amides being synthesised containing pharmaceutically relevant heterocycles, and difficulties encountered in the amidation of simple carbamate-protected amino acids ${ }^{28}$. Whilst there are examples of 
multigram scale reactions using simple boronic acid catalysts ${ }^{34-35}$, most are run on very small scale and show very poor reaction efficiency in terms of PMI ( 200-500). Chlorinated catecholborane derivatives were reported to show high catalytic efficiency with more hindered carboxylic acids and amines, but the reaction scope has yet to be explored in detail, and optimised scale-up reactions were not described ${ }^{29}$. A very recent report ${ }^{30}$ describes relatively complex BNB heterocyclic catalysts which show a broad substrate scope with regard to heterocyclic compounds and amino acid derivatives, but few reactions with less nucleophilic amines were reported. It is not clear yet whether these reactions can be scaled effectively (largest scale reported $1 \mathrm{~g}$ ), and if they offer advantages that can outweigh the increased synthetic effort required to prepare the catalyst.

a)

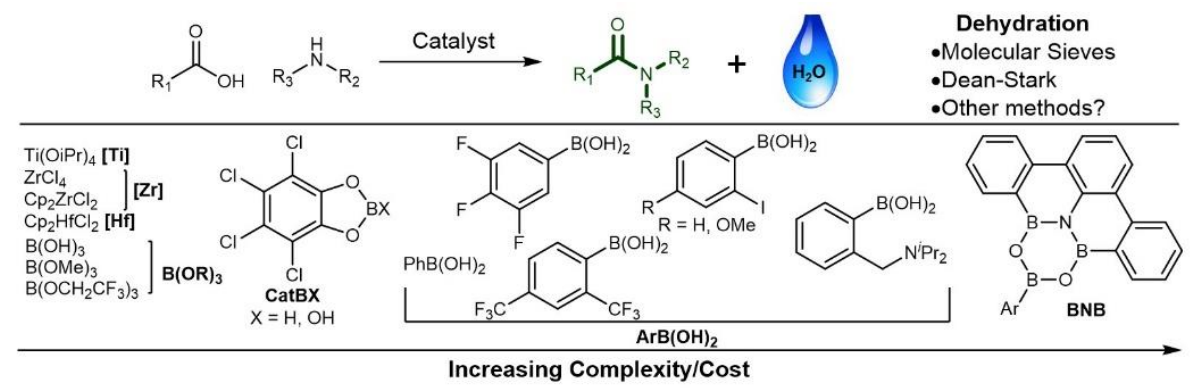

b)

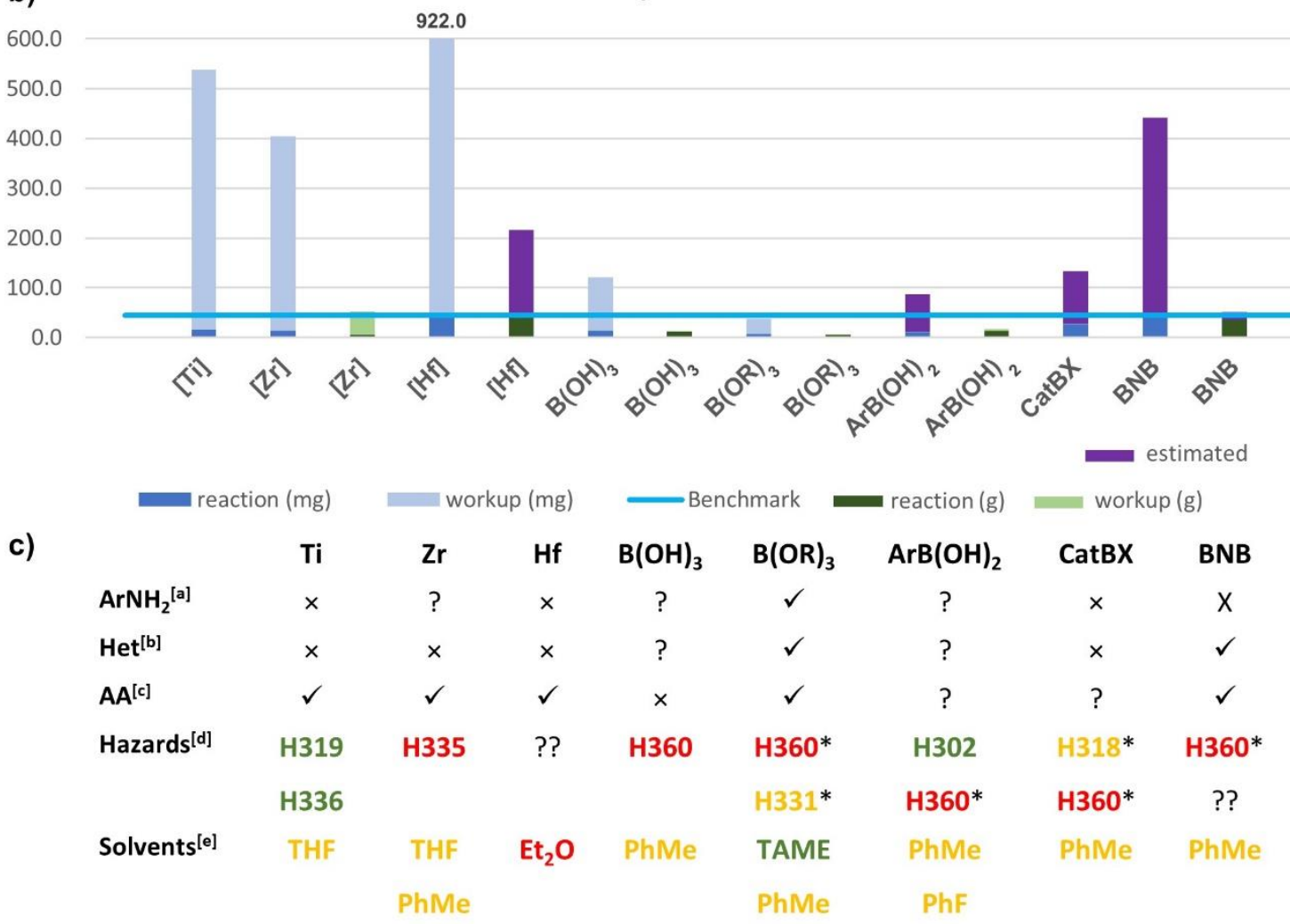

Figure 3: Catalytic direct amidation reactions a) Approaches to catalytic direct amidation; b) PMI data for milligram (blue) and gram scale (green) catalytic direct amidation reactions excluding purification (benchmark PMI 45); Estimated PMI data (purple) for reactions where 
solvent volume information is not provided in the literature. c) Further reaction data: Reaction scope (catalyst can be used for amidation of: ${ }^{[a]}$ Electron-deficient anilines; ${ }^{[b]}$ Heterocyclic substrates; ${ }^{[c]}$ Carbamate-protected amino acids; $\checkmark=$ Yes, $x=$ No/no examples reported; ?=limited data); [d]Most problematic health hazards on the MSDS for the catalyst ["*' indicates health hazards of breakdown products: $\mathrm{B}\left(\mathrm{OCH}_{2} \mathrm{CF}_{3}\right)_{3}-\mathrm{B}(\mathrm{OH})_{3}$ and $\mathrm{CF}_{3} \mathrm{CH}_{2} \mathrm{OH} ; \mathrm{ArB}(\mathrm{OH})_{2}-$

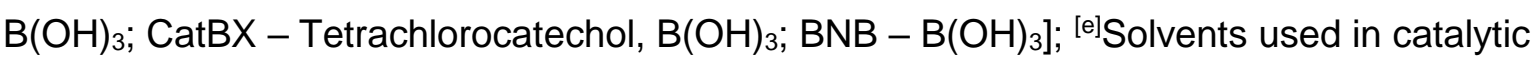
amidation reactions (colours refer to $\mathrm{CHEM}_{21}$ solvent guide ${ }^{9}$; Green=recommended; Orange=problematic; Red=hazardous; PhF is not included in the CHEM21 solvent guide, but can be considered as relatively non-hazardous for a halogenated solvent, but potentially problematic from an environmental perspective).

All of the catalytic amidation reactions in Figure 3 can be performed in solvents that are suitable for scale-up (PhMe, TAME, THF), though only TAME is listed as 'recommended' in a recent solvent guide ${ }^{9}$, with $\mathrm{PhMe}$ and THF described as 'problematic' and $\mathrm{Et}_{2} \mathrm{O}$ as 'hazardous'. It would therefore be useful to explore whether less-hazardous solvents can also be employed (e.g. esters ${ }^{35}$ ) in these reactions. By comparison with the PMI benchmark for a typical large-scale amidation performed within GSK (cyan line, PMI=45), it is clear that some of these catalytic methods can be very efficient, with boric acid, borate esters, and simple boronic acids being particularly suitable for scale-up. Of these, borate esters have a greater reported substrate scope in terms of functionalised acids/amines and have been demonstrated to be particularly efficient on gram scale; boric acid and boronic acids have been demonstrated on kilo scale, however. For the other boron catalysts and group(IV) metal catalysts, further work needs to be done to demonstrate the efficient scale-up of reactions and their application to pharmaceutically relevant substrates.

\section{Alternative approaches to amides}

Whilst direct condensation between a carboxylic acid and an amine presents the most straightforward and versatile approach to an amide, catalytic methods employing alternatives to carboxylic acids have also been described (Figure 4). In general, fewer of these starting materials are commercially available, in comparison to the vast range of available carboxylic acids, but these approaches can nevertheless prove advantageous for accessing particular classes of amides. 


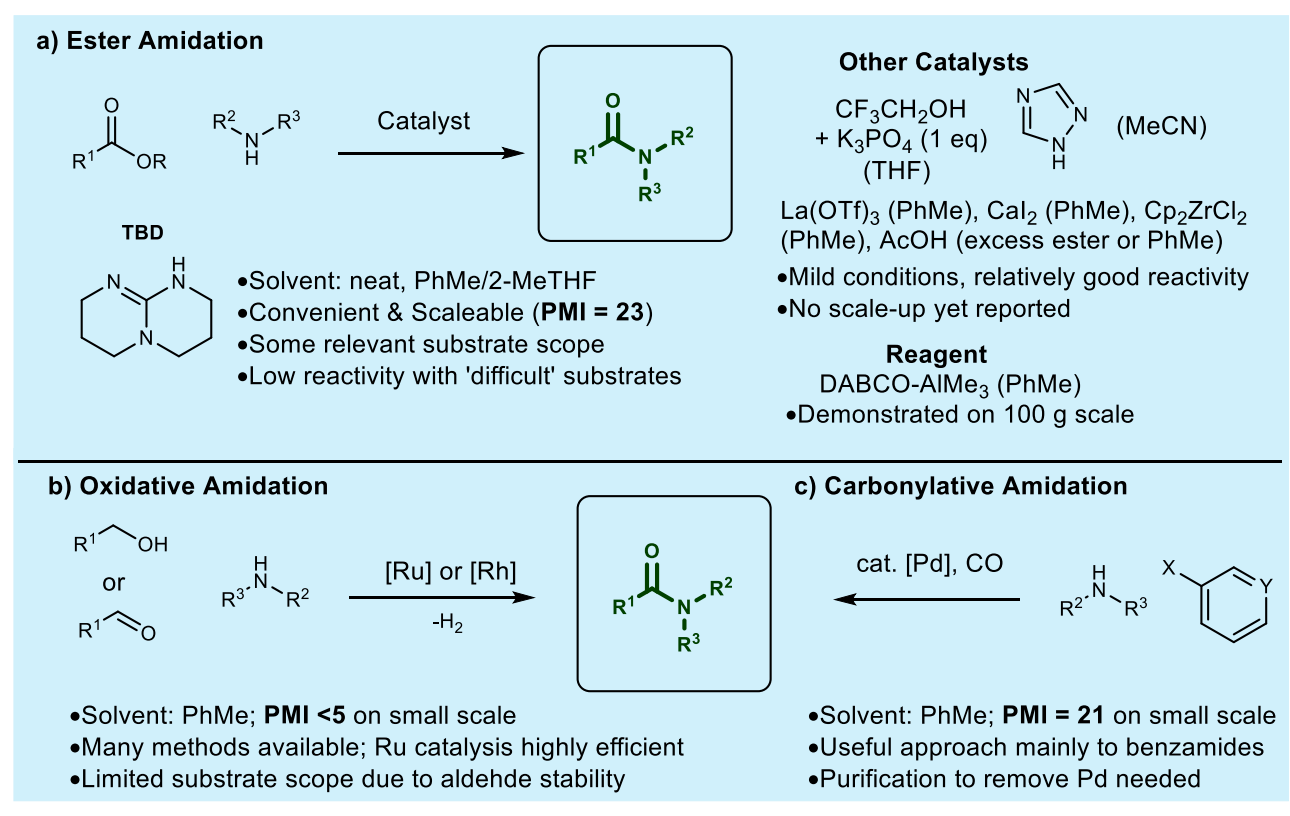

Figure 4: Alternative catalytic amidation approaches a) Amidation of unactivated esters; b) Oxidative amidation of alcohols/aldehydes; c) Carbonylative amidation of aryl halides. Typical solvents are shown in parentheses for each class of reaction, along with PMI data for selected reactions (excluding purification) in bold.

Ester amidation. A potentially useful alternative to the direct condensation of carboxylic acids and amines is the amidation of an ester with an amine (Figure 4a). Whilst activated esters are well known to undergo aminolysis under mild conditions, simple unactivated esters (methyl, ethyl) are essentially unreactive towards most amines except under forcing conditions. However, a number of catalytic methods are available to achieve direct aminolysis of these classes of unactivated ester, and reagents such as the air-stable DABCO-AIMe ${ }_{3}$ complex ${ }^{36}$ can also be employed. This can be a particularly attractive option in pharmaceutical synthesis, as amidation steps are often carried out on relative late-stage intermediates that will usually have been prepared with the carboxyl function protected as an ester. A wide range of catalysts have been reported in this area, but only a few methods have been demonstrated on large-scale. The use of TBD as an organocatalyst for direct amidation of esters ${ }^{37}$ remains attractive even though catalyst loadings are often high and the substrate scope remains relatively limited. This process was applied on kilogram scale for the synthesis of an HPGDS inhibitor in high purity $(P M I=23)^{38}$. Related approaches using other nitrogen nucleophiles such as triazole are also interesting ${ }^{39}$, though at the time of writing no exploration of their use on large scale has been reported. The use of trifluoroethanol to generate a reactive ester in situ via transesterification was reported by Watson \& Jamieson ${ }^{40}$, and this mild protocol can be applied to a range of substrates, though a stoichiometric quantity of base is required, and large-scale reactions were not described. 
Other reported catalysts for ester amidation include $\mathrm{Cp}_{2} \mathrm{ZrCl}_{2}{ }^{41}, \mathrm{Cal}_{2}{ }^{42}, \mathrm{La}(\mathrm{OTf})_{3}{ }^{43}$, and acetic acid $^{44}$. The latter is particularly interesting from a safety/sustainability perspective but it has yet to be widely explored.

Oxidative amidation. By using an oxidative reaction process, a hemiaminal, generated by in situ reaction of an amine and an aldehyde, can be converted into an amide ${ }^{45}$. Whilst most reactions of this type employ a stoichiometric oxidant, Milstein and co-workers described a method using a ruthenium catalyst that can oxidise the hemiaminal with release of hydrogen gas $^{46}$. Furthermore, the catalyst can also oxidise a primary alcohol to generate the required aldehyde in situ. Overall the process is highly atom-efficient, with the only stoichiometric byproduct being two molecules of hydrogen, and it can proceed with low catalyst loadings and small quantities of solvent. This leads to a reaction with a very low $\mathrm{PMI}(<5$, excluding purification), that is potentially scalable, although to the best of our knowledge there are no reports of this reaction being used on large scale for the preparation of pharmaceutical intermediates. The main limitation of this approach is the relatively limited substrate scope, with no detailed exploration of the reaction with pharmaceutically relevant functionalised compounds.

Carbonylative amidation. Palladium-catalysed carbonylative amidation of aryl halides provides another alternative approach to benzamides, which makes use of widespread expertise in Pd-catalysed chemistry, and the relative abundance of commercially available aryl halides ${ }^{47}$. Whilst this approach is largely limited to aryl and heteroaryl amides, these classes of amide are widely prevalent in pharmaceutically relevant substances. This process uses inherently more expensive catalysts but at lower loadings (in comparison to catalytic direct amidation of carboxylic acids), and leads to the generation of more stoichiometric waste products (e.g. halide salts, base-derived waste; $\mathrm{PMI}=21$, excluding chromatography) than direct amidation of carboxylic acids/esters.

Biocatalysis \& Heterogeneous Catalysis. Whilst still at a somewhat preliminary stage, biocatalysis offers another potential solution for sustainable amide formation. Examples of biocatalytic direct amidation of both carboxylic acids and esters have been reported ${ }^{48}$, and other biocatalytic reactions have often proved to be highly competitive for scale-up synthesis of pharmaceuticals in terms of efficiency and sustainability. Considerable work needs to be done to improve the range of enzymes available and their reaction scope to make them applicable to large-scale synthesis of complex amides, but this approach is almost certain to become more commonplace in the future as more effective biocatalysts are developed. In a similar vein, heterogeneous catalysts such as silica gel have been demonstrated to be highly 
efficient for direct amidation ${ }^{49}$, and have also been used in flow systems. ${ }^{50}$ As yet the scope with regard to synthesis of functionalised amides remains limited, however.

\section{Summary \& outlook}

Despite the fact that the first catalytic amidation of carboxylic acids was reported more than 20 years ago, and that a plethora of new methods have been reported in the past decade, the use of these processes in a preparative context on any significant scale is almost nonexistent. Within most research laboratories, amide synthesis is invariably achieved using a common stoichiometric coupling reagent. Partly, this is a matter of convenience, as most laboratories have these reagents already at hand, and they work well for most amides. Conversely, many catalytic amidation reactions are more difficult to set-up (e.g. activation of molecular sieves) and their suitability for preparing the functionalised amides researchers typically want to make is often uncertain. In terms of the preparation of large-scale quantities of drug candidate molecules, most catalytic amidation reactions fall short in terms of substrate scope, as well as efficiency and sustainability, in comparison to existing approaches involving stoichiometric activation. However, there are a few examples of boroncatalysed amidation reactions on large scale (boric acid ${ }^{33-34}$, boronic acid $^{34-35}$ ), and catalytic ester amidation has also been employed ${ }^{38}$. Nevertheless, the low uptake of catalytic amidation by the wider chemistry community is disappointing, because it illustrates that researchers developing new amidation methods have often failed to take account of the driving forces promoting interest in this area - i.e. a catalytic amidation should lead to less waste, and a more efficient and sustainable process overall if it is to become widely adopted. Whilst catalytic amidation reactions are starting to demonstrate considerable potential as alternatives to stoichiometric coupling reagents, very little work has been published that specifically addresses the optimisation and scale-up of these reactions for improved sustainability and efficiency. Given that most academic researchers emphasise the industrial importance of amide synthesis and the poor efficiency of coupling reagents when reporting new amidation methods, it is somewhat unfortunate that they often fail to consider the degree to which their own work addresses these issues. We would encourage all researchers working in this area to demonstrate that new catalytic amidation processes are scalable (at least to 10-20 g) scale, and to calculate the associated PMI for a preparative reaction and work-up, in order to assess the efficiency of the process. It is also of importance to assess the solvent scope and functional group tolerance of these reactions - after all, few chemists actually want to prepare simple unfunctionalized amides, so the scope (and limitations) of the reaction with regard to complex functionalised/heterocyclic substrates is of considerable interest ${ }^{51}$. We hope that by providing a concise overview of the current state-ofthe-art in catalytic amidation reactions, the chemistry community can be encouraged to 
become a little more adventurous when it comes to amide preparation. We also hope that future developments in this area will begin to address the remaining challenges preventing widespread adoption of catalytic amidation methods.

Acknowledgements: M.T.S. would like to acknowledge financial support for a postdoctoral position from UCL via the EPSRC Impact Acceleration Account (EP/R511638/1), and for a PhD studentship from UCL and GSK. L.T.B and H.F.S would like to thank K. Wheelhouse (GSK) for initial work on GSK portfolio reaction classification.

Author Contributions: M.T.S. and T.D.S wrote the manuscript and collected and analysed the Reaxys dataset, L.T.B \& H.F.S. collected and analysed the large-scale amidation dataset and contributed to the writing of the manuscript.

Competing Interests: The authors have no competing interests to declare.

Data Availability Statement: All data analysed in this article are included in the supplementary information file.

\footnotetext{
1 Valeur, E. \& Bradley M. Amide bond formation: beyond the myth of coupling reagents. Chem. Soc. Rev. 38, 606-631 (2009).

${ }^{2}$ Dunetz, J. R. Magano, J. \& Weisenburger, G. A. Large-Scale Applications of Amide Coupling Reagents for the Synthesis of Pharmaceuticals. Org. Process Res. Dev. 20, 140-177 (2016)

${ }^{3}$ Anastas, P. T.; Warner, J. C. Green Chemistry: Theory and Practice, Oxford University Press: New York. (1998).

${ }^{4}$ Jimenez-Gonzalez, C. et al. Using the right green yardstick: Why process mass intensity is used in the pharmaceutical industry to drive more sustainable processes. Org. Process Res. Dev. 15, 912 917 (2011).

${ }^{5}$ Constable, D. J. C. et al. Key green chemistry research areas-a perspective from pharmaceutical manufacturers. Green Chem. 9, 411-420 (2007).

${ }^{6}$ Brown, D. G. \& Boström, J. Analysis of Past and Present Synthetic Methodologies on Medicinal Chemistry: Where Have All the New Reactions Gone? J. Med. Chem. 59, 4443-4458 (2016).

${ }^{7}$ Sherwood, J., Farmer, T. J. \& Clark, J. H. Catalyst: Possible Consequences of the N-Methyl Pyrrolidone REACH Restriction. Chem 4, 2010-2012 (2018).

8 Sherwood, J. The European 1,2-Dichloroethane Ban Should Liberate not Limit C-H Activation Research and Development. Angew. Chem. Int. Ed. In press doi: 10.1002/anie.201800549 (2018). 9 Prat, D. et al. CHEM21 selection guide of classical- and less classical-solvents. Green Chem. 18, 288-296 (2016).

10 Li, C.-J. * Trost, B. M. Green chemistry for chemical synthesis. Proc. Natl. Acad. Sci. 105, $13197-$ 13202 (2008).

11 Ishihara, K. Ohara, S. \& Yamamoto, H. 3,4,5-Trifluorobenzeneboronic Acid as an Extremely Active Amidation Catalyst. J. Org. Chem. 61, 4196-4197 (1996).

12 MacMillan, D. S. et al. Evaluation of alternative solvents in common amide coupling reactions: replacement of dichloromethane and N,N-dimethylformamide. Green Chem. 15, 596-600 (2013).

${ }_{13} \mathrm{Ho}$, G.-J. et al. Carbodiimide-Mediated Amide Formation in a Two-Phase System. A High-Yield and Low-Racemization Procedure for Peptide Synthesis. J. Org. Chem. 60, 3569-3570 (1995).

${ }^{14}$ Carey, J. S. et al. Analysis of the reactions used for the preparation of drug candidate molecules. Org. Biomol. Chem. 4, 2337-2347 (2006).

${ }^{15}$ Braddock, D. C. et al. Tetramethyl Orthosilicate (TMOS) as a Reagent for Direct Amidation of Carboxylic Acids. Org. Lett. 20, 950-953 (2018).
} 
${ }^{16}$ Gabriel, C. M. et al. Amide and Peptide Bond Formation in Water at Room Temperature. Org. Lett. 17, 3968-3971 (2015).

17 Wehrstedt, K. D. Wandrey, P. A. \& Heitkamp, D. Explosive properties of 1-hydroxybenzotriazoles.

J. Hazard. Mater. 126, 1-7 (2005).

${ }^{18}$ Allen, C. L. Chhatwal, A. R. \& Williams, J. M. J. Direct amide formation from unactivated carboxylic acids and amines. Chem. Commun. 48, 666-668 (2012).

${ }^{19}$ Lundberg, H. Tinnis, F. \& Adolfsson H. Direct Amide Coupling of Non-activated Carboxylic Acids and Amines Catalysed by Zirconium(IV) Chloride. Chem. Eur. J. 18, 3822-3826 (2012).

${ }^{20}$ Lundberg, H. Tinnis, F. \& Adolfsson H. Titanium(IV) Isopropoxide as an Efficient Catalyst for Direct Amidation of Nonactivated Carboxylic Acids. Synlett 2201-2204 (2012).

${ }^{21}$ Lundberg, H. \& Adolfsson H. Hafnium-Catalyzed Direct Amide Formation at Room Temperature. ACS Catal. 5, 3271-3277 (2015).

22 Tang, P. Boric Acid Catalyzed Amide Formation from Carboxylic Acids and Amines: N-Benzyl-4phenylbutyramide. Org. Synth. 81, 262-272 (2005).

${ }^{23}$ Sabatini, M. T. Boulton, L. T \& Sheppard, T. D. Borate esters: Simple catalysts for the sustainable synthesis of complex amides. Sci Adv. 3, e1701028 (2017).

${ }^{24}$ Sabatini, M. T. et al. Protecting-Group-Free Amidation of Amino Acids using Lewis Acid Catalysts. Chem. Eur. J. 24, 7033-7043 (2018).

${ }^{25}$ Arnold, K. et al. Synthesis, evaluation and application of novel bifunctional N,N-

diisopropylbenzylamineboronic acid catalysts for direct amide formation between carboxylic acids and amines. Green Chem. 10, 124-134 (2008).

${ }^{26}$ Fatemi, S. Gernignon, N. \& Hall, D. G. A multigram-scale lower E-factor procedure for MIBAcatalyzed direct amidation and its application to the coupling of alpha and beta aminoacids. Green Chem. 17, 4016-4028 (2015).

${ }^{27}$ Wang, K. Lu, Y. \& Ishihara, K. The ortho-substituent on 2,4-bis(trifluoromethyl)phenylboronic acid catalyzed dehydrative condensation between carboxylic acids and amines. Chem. Commun. 54, 5410-5413 (2018).

${ }^{28}$ Fatemi, S. Gernigon, Hall, D. G. A multigram-scale lower E-factor procedure for MIBA-catalyzed direct amidation and its application to the coupling of alpha and beta aminoacids. Green Chem. 17, 4016-4028 (2015).

${ }^{29}$ Maki, T. Ishihara, K. \& Yamamoto, H. 4,5,6,7-Tetrachlorobenzo[d][1,3,2]dioxaborol-2-ol as an effective catalyst for the amide condensation of sterically demanding carboxylic acids. Org. Lett. 8, 1431-1434 (2006).

${ }_{30} \mathrm{Noda}, \mathrm{H}$. et al. Unique physicochemical and catalytic properties dictated by the $\mathrm{B}_{3} \mathrm{NO}_{2}$ ring system. Nature Chem. 9, 571-577 (2017).

31 https://www.bgs.ac.uk/mineralsuk/statistics/risklist.html.

32 Moore, J. A. et al. An Assessment of Boric Acid and Borax using the IEHR Evaluative Process for Assessing Human Developmental and Reproductive Toxicity of Agents. Reproductive Toxicol. 11, 123-160 (1997).

${ }^{33}$ Bannister, R. B. et al. A Scaleable Route to the Pure Enantiomers of Verapamil. Org. Process Res. Dev. 4, 467-472 (2000).

${ }^{34}$ Mylavarapu, R. K. et al. Boric Acid Catalyzed Amidation in the Synthesis of Active Pharmaceutical Ingredients. Org. Process Res. Dev. 11, 1065-1068 (2007).

${ }^{35}$ Limanto, J. et al. A Highly Efficient Asymmetric Synthesis of Vernakalant. Org. Lett. 16, 2716-2719 (2014).

${ }^{36}$ Lee, D. S. et al. Investigating Scale-Up and Further Applications of DABAL-Me 3 Promoted Amide Synthesis. Org. Process Res. Dev. 19, 831-840 (2015).

${ }^{37}$ Sabot, C. et al. A convenient aminolysis of esters catalyzed by 1,5,7-triazabicyclo[4.4.0]dec-5-ene (TBD) under solvent-free conditions. Tetrahedron Lett. 48, 3863-3866 (2007).

38 Weiberth, F. J. et al. Demonstration on Pilot-Plant Scale of the Utility of 1,5,7-

Triazabicyclo[4.4.0]dec-5-ene (TBD) as a Catalyst in the Efficient Amidation of an Unactivated Methyl Ester. Org. Process Res. Dev. 16, 1967-1969 (2012).

39 Yang, X. \& Birman, V. B. Acyl Transfer Catalysis with 1,2,4-Triazole Anion. Org. Lett. 11, 14991502 (2009).

40 McPharson, C. G. et al. Amidation of Unactivated Ester Derivatives Mediated by Trifluoroethanol. Org. Biomol. Chem. 15, 3507-3518 (2017).

41 Lenstra, D. C. Nguyen, D. T. \& Mecinović, J. Zirconium-catalyzed direct amide bond formation between carboxylic esters and amines. Tetrahedron Lett. 71, 5547-5553 (2015).

42 Nguyen, D. T. Lenstra, D. C. \& Mecinović, J. Chemoselective calcium-catalysed direct amidation of carboxylic esters. RSC Adv. 5, 77658-77661 (2015). 
${ }^{43}$ Morimoto, H. et al. Lanthanum(III) Triflate Catalyzed Direct Amidation of Esters. Org. Lett. 16, 20182021 (2014).

${ }^{44}$ Sanz Sharley, D. D. \& Williams, J. M. J. Acetic acid as a catalyst for the $\mathrm{N}$-acylation of amines using esters as the acyl source. Chem. Commun. 53, 2020-2023 (2017).

45 Tillack, A. Rudloff, I. \& Beller, M. Catalytic Amination of Aldehydes to Amides. Eur. J. Org. Chem. 2001, 523-528 (2001).

${ }^{26}$ Gunanathan, C. Ben-David, Y. \& Milstein, D. Direct Synthesis of Amides from Alcohols and Amines with Liberation of $\mathrm{H}_{2}$. Science 317, 790-792 (2007).

${ }^{47}$ Fang, W. et al. Highly Efficient Aminocarbonylation of lodoarenes at Atmospheric Pressure Catalyzed by a Robust Acenaphthoimidazolyidene Allylic Palladium Complex. Org. Lett. 15, 36783681 (2013).

${ }^{48}$ Dorr, B. M. \& Fuerst, D. E. Enzymatic amidation for industrial applications. Curr. Opin. Chem. Bio. 43, 127-133 (2018).

${ }^{49}$ Comerford, J. W. et al. Clean, reusable and low cost heterogeneous catalyst for amide synthesis. Chem. Commun. 2562-2564 (2009).

${ }^{50}$ Petchey, T. H. M. et al. Optimization of Amidation Reactions Using Predictive Tools for the Replacement of Regulated Solvents with Safer Biobased Alternatives. ACS Sus. Chem. Eng. 6, 15501554 (2018).

${ }^{51}$ Nadin, A. Hattotuwagama, C. \& Churcher, I. Lead-Oriented Synthesis: A New Opportunity for Synthetic Chemistry. Angew. Chem. Int. Ed. 51, 1114-1122 (2012). 